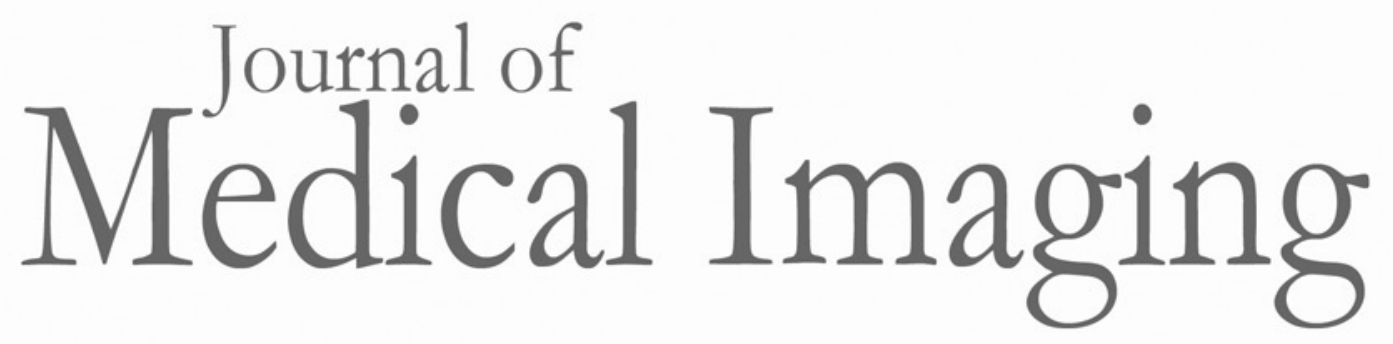

Medicallmaging.SPIEDigitalLibrary.org

\title{
Special historical reprint: An assortment of image quality indexes for radiographic film-screen combinations-can they be resolved?
}

Kyle Myers

Weijie Chen

\section{SPIE.}




\title{
Special historical reprint: An assortment of image quality indexes for radiographic film-screen combinations-can they be resolved?
}

\author{
Kyle Myers, Guest Editor \\ Weijie Chen, Guest Editor \\ U.S. Food and Drug Administration
}

\begin{abstract}
Robert F. Wagner wrote his first SPIE paper ${ }^{1}$ in 1972, within the first year of his joining the Bureau of Radiological Health, the precursor to the FDA's Center for Devices and Radiological Health. He had been hired to build a laboratory and develop methodologies for assessing the performance of diagnostic x-ray systems, in support of the passage of the Radiation Control for Health and Safety Act. In that first year, Bob met with leading scientists in medical imaging as well as other specialties including vision, communications, and television. He formulated a risk-benefit approach to his work, recognizing that the patient exposure associated with the creation of a medical image needed to be considered in light of the usefulness of that image. Bob's manuscript, reprinted in this special section of the Journal of Medical Imaging, provided an insightful review of the image quantification field, including modulation transfer functions, Wiener spectra, and the basis for receiver operating characteristic curves, along with a bold statement that laid the foundation for the entire field of medical imaging assessment to follow, that image quality "must be defined in terms of the task that the image is destined to perform." $\odot 2014$ Society of Photo-Optical Instrumentation Engineers (SPIE) [DOI: 10.1117/1.JMI.1.3.031013]
\end{abstract}

\section{References}

1. R. F. Wagner and K. E. Weaver, "An assortment of image quality indexes for radiographic film-screen combinations-can they be resolved?," Proc. SPIE 0035, 83-95 (1972). 


\title{
AN ASSORTMENT OF IMAGE QUALITY INDEXES FOR RADIOGRAPHIC FIIM-SCREEN COMBINATIONS --- CAN THEY BE RESOLVED?
}

\author{
Robert F. Wagner and Kenneth E. Weaver \\ Bureau of Radiological Health \\ Food and Drug Administration \\ Rockville, Maryland
}

\begin{abstract}
PREFACE
The following briefly summarizes the background on how the Bureau of Radiological Health became involved in the image analysis area. In October 1968 the Radiation Control for Health and Safety Act, Public Law 90-602, gave the Secretary of the Department of Health, Education, and Welfare and by delegation to the Food and Drug Administration new functions involving the radiation safety responsibility to control ionizing and nonionizing radiation emitted from electronic products. A majority of the responsibilities under the law have been redelegated to the Bureau of Radiological Health.
\end{abstract}

To date four radiation safety performance standards have been promulgated under the law; these pertain to television receivers, microwave ovens, demonstration type coldcathode gas discharge tubes and medical and dental diagnostic $\mathrm{x}$-ray systems.

The diagnostic $x$-ray standard became effective when published in the Federal Register on August 15, 1972. It will apply to all such products manufactured after August 15, 1973. An integral part of the diagnostic $x$-ray system which was not addressed in this standard is the image receptor system (film-screen combinations, grids, cassettes, etc.) and the associated film processing. Therefore, we are developing laboratory capabilities and methodology in order to assess and test the performance of these items as a prerequisite to possible inclusion of expanded performance requirements under the diagnostic $\mathrm{x}$-ray standard.

Moreover, the Bureau has traditionally assumed a role of encouraging and promoting procedures, devices, techniques, and use of equipment which result in the reduction of patient exposure and/or improved diagnosis. To this end, we are particularly interested in evaluating screens made from newly reported phosphors (Ref. 1, 2) which show promise for significantly reducing patient exposure while possibly yielding image quality commensurate with or better than present screens.

During the stage of preparation for this study we have been procuring and fabricating equipment for development of an image analysis laboratory, consulting with experts in the field, and carrying out a survey of some techniques for quantifying image quality. We have tried to learn how much of the problem of image analysis has been solved and to find where difficulties remain. The following includes a description of our plans, the results of our survey, and some suggestions for the on-going dialogue concerning this interesting question.

\section{INTRODUCTION}

The question of image quality has been an elusive one to define. One thing, however, is certain - that it must be defined in terms of the task that the image is destined to perform. In our context the task is the presentation of diagnostic information (generally low contrast, and otherwise difficult to define) to the eye-brain system of an individual with a highly specialized collection of experience, viz., the radiologist. He is the ultimate judge of image quality. For several decades now, however, techniques from communications and optical engineering have been used to aid him in selecting imaging systems. Drs. Russell H. Morgan and Kurt Rossmann have introduced some very successful applications of these techniques to the problem of predicting which systems are best suited to certain particular tasks in Radiology. Their measurements and methods of system analysis have been complementary to the methods of the community of Radiologists. It is in the spirit of their investigations that this review is written; that is, how to quantify radiological imaging systems in a way that makes the engineering parameters explicit and yields some prediction (even though loose and provisional) as to their imaging capability. 


\section{SYSTEM PARAMETERS}

Certain parameters can be measured which characterize the imaging system. These include the sensitometry of the screen-film processing combination, the modulation transfer function and the Wiener spectrum of the system noise.

\section{$\underline{\text { Sensitometry }}$}

The $\mathrm{H}$ and $\mathrm{D}$ curve (density versus log $\mathrm{x}$-ray exposure of the film-screen processing combination) can be determined by either using intensity-scale sensitometry (holding exposure time constant and varying the $x$-ray intensity) or time-scale sensitometry (holding $x$-ray intensity constant and varying the exposure time.) When radiographic film is used with intensifying screens it is mainly exposed by light from the screens. In this case timescale and intensity scale sensitometry will, in general, result in different characteristic curves due to reciprocity law failure. Intensity-scale sensitometry rather than time scale sensitometry will be used since it most nearly simulates the actual conditions under which the screen film combination is exposed in radiography. Also, the technique for determining the line spread function of the screen is made with constant exposure time and thus derivation of the line spread function from the slit exposure density distribution requires the use of intensity-scale sensitometry. In addition to using the sensitometric curve in the derivation of the line spread function it will also be used to determine the speed (roentgens-l) of the filmscreen processing combination for a particular beam quality, and its contrast characteristics (such as the average gradient).

The $\mathrm{x}$-ray generating equipment that will be used for doing the sensitometric exposures is identical to that used by Bates (Ref. 3). Automation of the sensitometer will be patterned after a system designed by Haus (Ref.4).

MTF

Useful parameters describing the system transfer characteristics (for the special case of one-dimensional inputs) for $\mathrm{x}$-ray intensifying screens are the line spread-function (ISF) and its Fourier transform, the modulation transfer-function (MTF). The LSF is the relative illuminance distribution in the image plane resulting from a narrow slit exposure in the object plane. The LSF (Figure 1) and MTF (Figure 2) thus provide a measure of the image deterioration due to light diffusion in the screens and film.

Considering that any one-dimensional object can be represented as an infinite number of

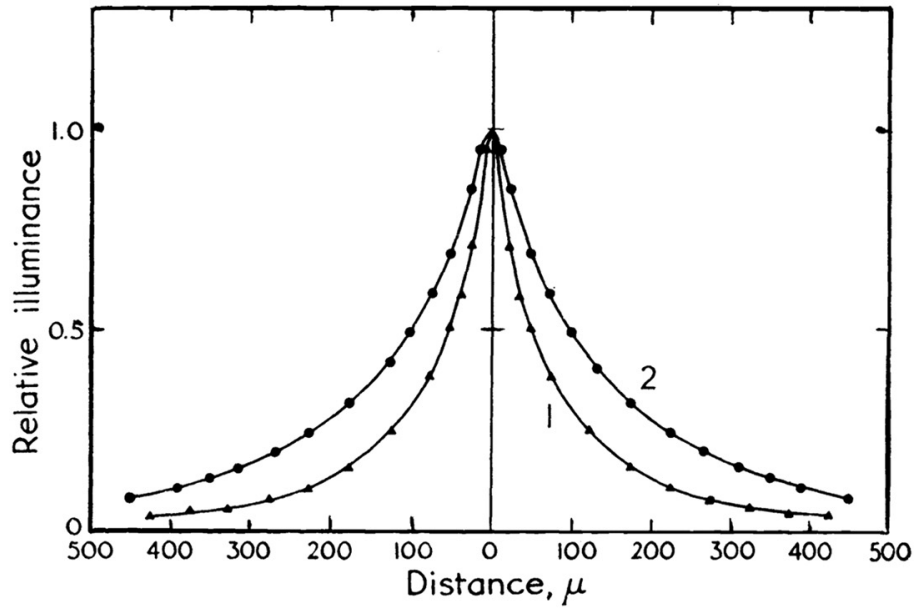

Figure 1. Normalized line spread-function of two radiographic systems containing calcium tungstate intensifying screens exposed with Kodak Royal Blue Film: (1) Two medium-speed screens; (2) Two fast screens. (Ref. 6l; courtesy of the Journal of Photographic Science).

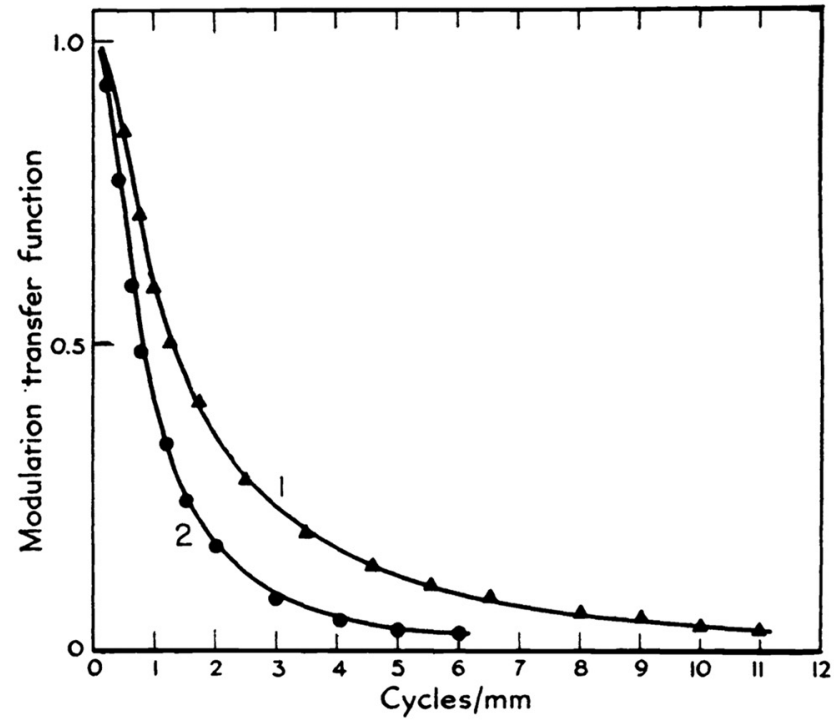

Figure 2. Modulation transfer functions calculated from the line spread-functions in Figure 1: (1) Two medium-speed screens; (2) Two fast screens. Both exposed with Kodak Royal Blue Film. (Ref. 61; courtesy of the Journal of Photographic Science).

line elements, from a measure of the radiation intensity distribution ( $x$-ray pattern) in the object plane and a knowledge of the system transfer characteristics, the resultant radiation intensity distribution in the image plane can be calculated. If the LSF is used to derive the output in the spatial domain, a convolution is performed 


$$
I_{\text {out }}(x)=\int_{-\infty}^{\infty} I_{\text {in }}(\xi) \cdot \operatorname{LSF}(x-\xi) d \xi
$$

where $I_{\text {out }}(\mathrm{x})$ and $I_{\text {in }}(\boldsymbol{\xi})$ are the output and input illuminances and $x$ and $\xi$ are coordinates in the image and object plane, respectively. The output in the frequency domain is determined by multiplication of the MTF, and the input spatial frequency spectrum.

$$
S_{\text {out }}(f)=M(f) \cdot S_{\text {in }}(f)
$$

where $S_{\text {out }}(f)$ and $S_{\text {in }}(f)$ are the output and input spatial frequency spectra.

As a check on the accuracy of this methodology, one can perform an edge exposure by removing one of the platinum jaws. It follows from equation (1) that the edge exposure distribution should be the integral of the slit exposure distribution (Figure 3, Ref. 5).

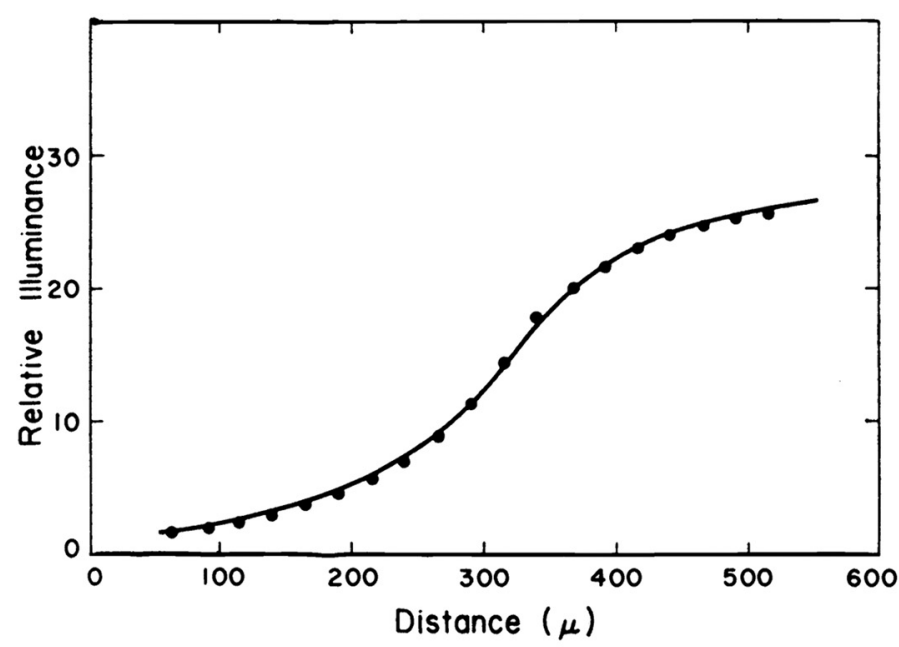

Figure 3. Intensity distribution in the radiographic image from an edge exposure. The curve is calculated directly from microdensitometer traces; the points are calculated by convolution of the edge with the line spreadfunction. (Ref. 5; courtesy of the Journal of the Optical Society of America).

Initially, our work will be limited to onedimensional analysis utilizing the MTF. To work with two-dimensional inputs requires knowledge of the point-spread-function PSF. There are certain difficulties associated with direct measurement of the quantity (Ref.6). However, Marchand (Ref. 7) has shown that for isotropic systems the PSF can be calculated from the ISF. Thus, two-dimensional analysis can be accomplished through additional labor, minimal for circularly symmetric objects.

The LSF of the screen-film combination will be determined by the slit method (Ref. 8, 9).
Briefly, the methodology consists of exposing the screen-film-system to $x$ rays through a 10-micron slit (the LSF generated is essentially identical to that produced by an infinitely narrow slit) formed by platinum jaws, $2 \mathrm{~mm}$ thick, and scanning the processed line image with a microdensitometer (the microdensitometer we have procured for this task is an Optronics Specscan S-3000).

The light output from the intensifying screen is directly proportional to the $x$-ray exposure input and the system is therefore linear (Ref. 6). However, the exposure response of the system is non-linear when the output is expressed in terms of optical density. Therefore, the characteristic curve of the screenfilm processing combination is used to calculate the relative exposure distribution (relative illuminance) from the line image density distribution obtained with the microdensitometer. This linearizes the system and yields the LSF. A digital computer (Hewlett Packard 2100A with a disc operating system) will be used to calculate the normalized LSF from the characteristic curve. The MTF is then calculated, utilizing the computer, from the normalized LSF by means of a Fourier transformation. Since the ISF is symmetrical a Fourier cosine transform is calculated.

\section{Wiener Spectrum}

The remaining characteristic of the filmscreen combination that we wish to measure is the system noise. In photographs this noise is called graininess and is due to the randomness of developed grains; it is generally white (constant spatial frequency spectral density) over the pass-band of the eye. In radiographs the noise is predominantly what is called "quantum mottle" and is due to the statistical fluctuations of absorbed x-ray quanta (Ref. 10, 11). This noise is generally highly correlated or colored: Its spectral density is much greater at the lower spatial frequencies of the pass-band of the eye and greatly attenuated at the higher frequencies due to the falloff in the response of the screen which transmits this noise (Ref. 11).

These fluctuations can be characterized by their spatial frequency power spectrum -- the so-called Wiener Spectrum N(f) (Ref. 12). This can be obtained from microdensitometer traces of film uniformly exposed to some average density by the intensifying screens. The variations in such traces have been analyzed by analog means by a number of workers in the photographic field. (Ref. 13, 14). One technique consists in coupling the output of a microdensitometer with a rotating table (containing the sample film) to an electronic frequency analyzer. Some typical results from the work of $K$. Doi are shown in Figure 4. 


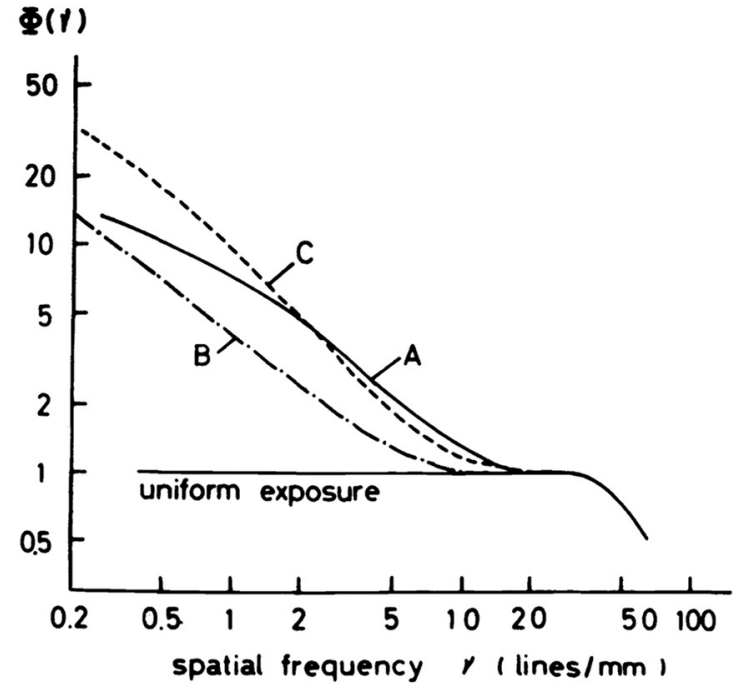

Figure 4. Wiener spectra of radiographic mottle (for three different speed screens A, $B$, and $C$ exposed with the same film type) and film graininess (Ref. 14; courtesy of Aesculapius Publishing Company).

The fluctuations in the traces can also be analyzed by digital techniques (Ref. 15) and by application of the theory of stationary time series (Ref. 16). This requires a very careful massaging of the data, since a given sample trace which provides an excellent statistical estimate of the mean and variance of the density fluctuations may give a very poor estimate of its spectral (spatial

frequency) character. This latter deficiency can be overcome by several techniques. One approach is to average many spectral estimates from an ensemble of traces. Another is to weight the data from a given trace with a so-called "data window". This is a multiplication in the spatial domain which results in a smearing or convolution in the frequency domain, that is, an averaging over neighboring spectral estimates. The averaging function is related to the window function through its Fourier transform. Figure 5 attempts to show this process schematically. We will apply a combination of these techniques and Fast Fourier methods described by Cooley et al. (Ref. 17). This combination minimizes the error in the spectral estimate for a given amount of data.

\section{INPUT PARAMETERS}

The parameters above will be used to characterize the imaging system. The imaging task will be further specified by the Fourier Spectra of some simple objects which might be considered characteristic of some typical radiographic tasks.

\section{HUMAN RECEPTOR PARAMETERS}

The final element in the analysis of the overall imaging system is the human eye-brain system. Any attempt to characterize this jack-in-the-black-box as an element to be cascaded with the input and imaging system must proceed with great care and limited expectations. We are dealing here with an enormously complicated non-linear information processing system. It has been known for some time that "the nervous system contains unique organizing principles as one of its inherent properties"; in fact, "All perceptions are recoded versions of an input which in principle cannot map 1:1 into a perception." (Ref. 18). For example, "the contours... and the size of the stimulus seem to be responded to directly by different parts of nervous systems; and these responses cannot be understood as reactions only to light flux. This discovery of stimulus-specific cells (sometimes called analyzers) is probably the most significant event in recent work in electrophysiology." (Ref. 19). This character of a special capacity for recognizing patterns is attended by interesting adjacency and nonlinear effects and hindered by the redundancy of too much information (Ref. 20, 2l).

In a word, we do not know how to solve the real system. As in many other complicated problems we proceed to solve a system which
Spatial Domain

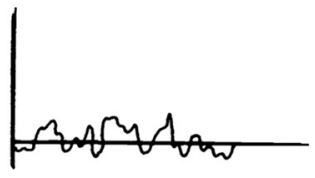

$\times$

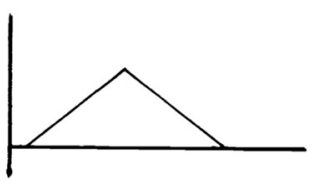

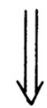

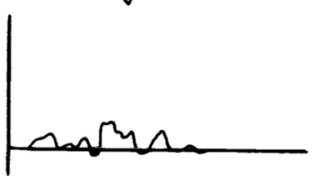

Power Spectrum Domain
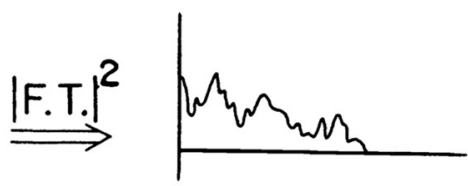

$\circledast$
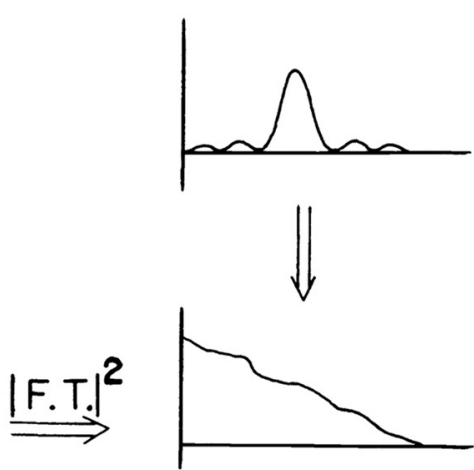

Figure 5. Smoothing of the spectral estimate -a convolution in the power spectmum domain -more easily achieved by viewing the data through an aperture of varying transmission (data window in the spatial domain. 
is solvable, and try to introduce modifications which bring it closer to a real system. This approach has led to the introduction of a transfer (describing) function $E(f)$ for the human visual system - a linear model that might have some utility for the low contrast situations encountered in radiography. Many such functions have been introduced (Ref. 2226). There is by no means widespread agreement on how to measure and interpret these functions. On two points there seems to be general agreement:(1) the response of the eye increases with frequency at the low spatial frequencies (the edge enhancement characteristic responsible for the Mach band effect) (Ref. 27); (2) the response of the eye falls off at high spatial frequencies (due to the dioptrics of the eye - a low pass filter) (Ref. 28). An example is given in Figure 6.

In this context of linear systems analysis we find a variety of expressions that have been used to quantify or predict image quality.

\section{IMAGE QUALITY INDEXES}

Non-Noisy Images

We begin by neglecting system noise. Several quality indexes for photographic systems emphasized sharpness through the mean square criterion. In this context, if the object is considered to have the structure of white Gaussian noise (principle of maximum ignoranceO'Neill (Ref. 29)) then Linfoot's index of relative structural content (Ref. 30) becomes

$$
T=\int M^{2}(f) d f=N_{e}
$$

i.e., this is the same as the quantity which Schade introduced for television systems -the equivalent pass band $\mathrm{N}_{e}(\operatorname{Ref} .31)$.

Higgins and Jones used the step function response or edge exposure as a basis for a photograpnic quality index (Ref. 32). It was found that the mean square gradient of the density distribution $\mathrm{dD} / \mathrm{dx}$ across an edge trace was a good objective correlate to the subjective impression of sharpness. Their "acuteness" index is written:

$$
A=\frac{1}{\Delta D}\left[\frac{1}{\Delta x} \int\left(\frac{d D}{d x}\right)^{2} d x\right]
$$

where, $\Delta \mathrm{x}, \Delta \mathrm{D}$ are the ranges of $\mathrm{x} \& \mathrm{D}$.

Roetling, Trabka and Kinzly have shown the connection between this and the equivalent pass band to be given by
TRANSFER FUNCTION OF COMPLETE VISUAL SYSTEM

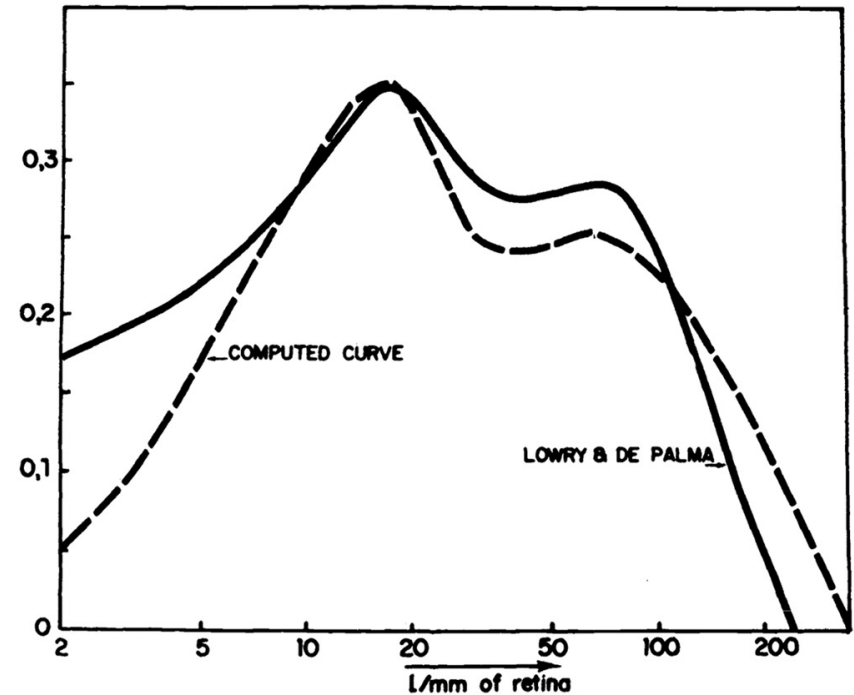

Figure 6. Transfer function of human visual system measured by Lowry and DePalma (Ref. 23), and a curve computed by DeBelder et al.

(Ref. 58), from their own model of the human visual system (Courtesy of Journal of Photographic Science).

$$
A \simeq \frac{2 \Delta D}{\Delta x} N_{e}
$$

for low contrast applications (Ref. 33).

The next step was to cascade the eye with the imaging system. The previous indexes for sharpness then become

$$
I \Rightarrow \int[M(f) \cdot E(f)]^{2} d f
$$

a quantity used by Schade (normalized to the passband of the eye and given on a logarithmic scale) and Ooue (Ref. 22, 34). Ooue tried other weightings (first or second power of either/both $M(f)$ and $E(f)$ but found only small differences among them as correlates of subjective sharpness.

Rao has pointed out that one must explicitly include the contrast of the film or screenfilm combination to obtain an objective measure of what he calls the Image Contrast Function (Ref. 35).

$$
\text { I.C.F. }=\gamma \cdot M(f) \quad \text { (non noisy images) }
$$

This expression has the same emphasis as the approximate expression for acuteness given above in equation (5), explicitly including the gain of the film (implicit in $\Delta D$ ). However, it does not penalize the system for spreading as severely as does acuteness. 
Finally, if one introduces the object spectrum $O(f)$ to specify the task, a quantity proportional to mean energy content or mean square contrast can be written

$$
\begin{aligned}
S & =\gamma^{2} \int[O(f) \cdot M(f) \cdot E(f)]^{2} d f \\
\text { or } S & =\gamma^{2} \int\left[M_{W}(f) \cdot E(f)\right]^{2} d f
\end{aligned}
$$

where we have written $\mathrm{M}_{\mathrm{w}}(f)$ for the system transfer function weighted by the object spectrum. This quantity has been used by Halmshaw (Ref. 36).

\section{Noise timited Images}

So far we have neglected the deterioration of image quality due to the presence of noise in the system. This is the colored quantum noise which we have discussed above. When noise is introduced our expressions above must be modified to

$$
I \Rightarrow a^{2}=\frac{S}{N}
$$

the ratio of signal energy to noise energy. The rest of this paper will concern the best way to calculate the signal-to-noise ratio. Its importance is already well-known from communication and information theory in one and two dimensions where it represents a measure of information, and from studies of perceptibility where it represents a measure of confidence.

For example, the information capacity of a channel of width $\Delta f$ is

$$
\text { (Ref. 37) } \quad c=\Delta f \log \left(1+\frac{S(f))}{N(f)}\right.
$$

If a system consists of many narrow subchannels, we may write for its total capacity

$$
C=\int \log \left(1+\frac{S(f)}{N(f)} d f\right.
$$

We are faced here with the problem of two extremes, vig., whether to be concerned with the ratio of total signal power to total noise power (if the eye is taken to be one very broad unselective channel) or to integrate the ratio over all relevant frequencies (if the eye contains a continuum of selective channels), of or course, something in between. We will return to this question shortly.
The relationship of signal-to-noise ratio to perceptibility was studied by Rose (Ref. 38.), and Coltman and Anderson (Ref.39). (For a review of this and related work see Ref. 40). A statistical model that incorporates many results of these studies of nonresolution limited objects has been suggested by Moran (Ref. 41). It predicts a sharp threshold effect in perception confidence versus signal-to-noise ratio (see Figure 7), with the threshold only weakly (logarithmically) dependent on the viewing conditions of object size, and viewing distance, and independent of object shape.

This work has been extended to include various image degrading effects and resolution limitations (Ref. 42, 43); but the solution of the general problem of arbitrary object and noise spectra is still evolving. We proceed to a discussion of some solutions which include the above studies as special cases.

\section{OPTIMUM DETECTORS}

How should one calculate the signal-to-noise ratio if he wishes to use it as an index of image quality for a system that includes a human observer? An approach that has been used for the auditory system and has been suggested for the visual system is to treat the organ-brain combination as some form of optimum detector. The first optimum detector we will consider is the matched filter, that is, a detector that is selectively tuned for maximum signal-to-noise for a particular input of interest (Ref. 44). For the case where the interfering noise is white, the eye that is so selectively tuned world have a transfer function

$$
|E(f)|=M_{W}(f)
$$

This detector is only troubled by that part of the noise spectrum that overlaps the signal spectrum, since that is the only region in which it responds. The maximum signal-tonoise ratio for such a detector is

$$
d_{I}^{2}=\frac{\gamma^{2} \int M_{w}^{2}(f) d f}{N_{0}}
$$

where $N_{O}=$ spectral density of noise (constant).

This quantity has been used to generate a figure of merit measuring picture resolution for complex systems (Ref. 45).

For the case where the interfering noise is solored, the selectively tuned detector 


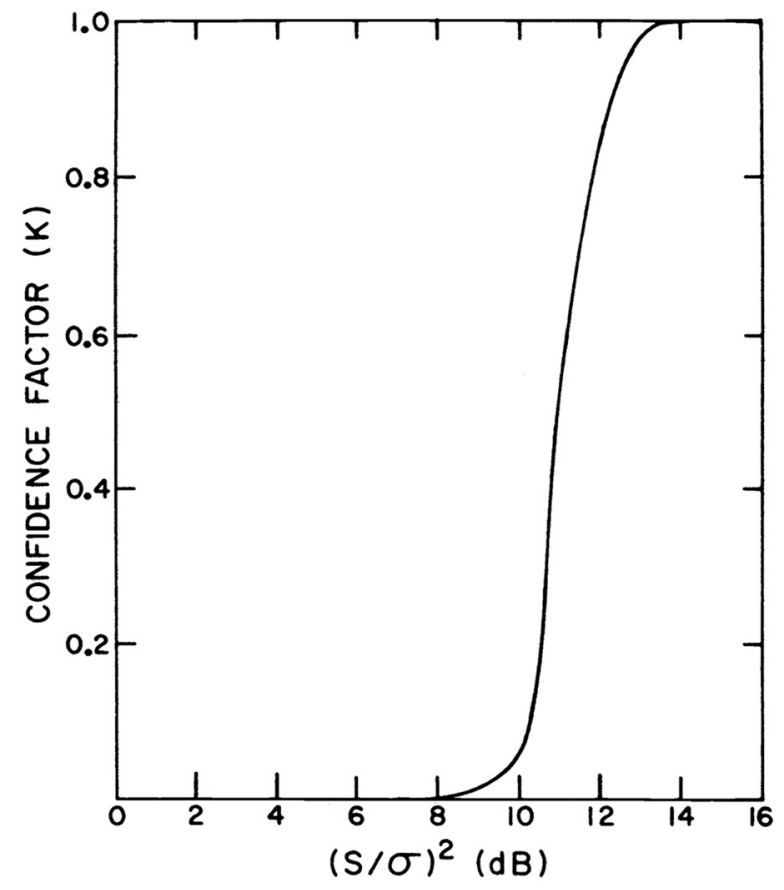

Figure 7. Confidence factor for object perception vs. signal-to-noise ratio as derived by P.R. Moran from his statistical model. The abscissa corresponds to $\mathrm{d}^{2}$ in the notation of the present article (Ref. 41; courtesy of P.R. Moran).

would have a transfer function

$$
|E(f)|=\frac{M_{W}(f)}{N(f)}
$$

that is, it is matched to the signal and against the noise.

The maximum signal-to-noise ratio for this detector is

$$
d_{2}^{2}=\gamma^{2} \int \frac{M_{w}^{2}(f)}{N(f)} d f
$$

Such a quantity has been suggested as a basis for evaluating image quality by Goodenough (Ref. 46), Lawson (Ref. 47), and in a modified form by Halmshaw (Ref. 36). Again, this detector is only troubled by noise where the noise and the image spectrum overlap.

Another version of an optimum detector, one that has been applied to the ear-brain system (Ref. 48) and the eye-brain system (Ref. 49), is the detector that bases its decision on the likelihood ratio. For the case of the "binary decision", this detector takes a set of readings and computes the likelihood ratio, that is, the ratio of the probability that its readings are due to the presence of the signal (in noise) to the probability that the readings are due to noise alone. The ratio is compared to a decision criterion. The detector responds that the signal is present (absent) if the computed ratio is greater (smaller) than some decision criterion. Harris (Ref. 49) treats the case of detecting a signal image in the presence of additive white, Gaussian noise. If the decision criterion is set equal to one, the probability of a true positive is

$$
P_{\text {T.P. }}=\frac{1}{(2 \pi)^{1 / 2}} \int_{-d / 2}^{\infty} e^{-z^{2} / 2} d z
$$

and the probability of a false positive (false alarm)is

$$
P_{\text {F.P. }}=\frac{1}{(2 \pi)^{l / 2}} \int_{d / 2}^{\infty} e^{-z^{2} / 2} d z
$$

where

$$
d^{2}=d_{1}^{2}=\frac{\gamma^{2} \int M_{w}^{2}(f) d f}{N_{0}}
$$

The signal-to-noise ratio is the same as that encountered for the case of the matched filter for white noise, equation (13). It has been noted elsewhere that the matched filter and the likelihood function detector are equivalent for this case (Ref. 50).

The likelihood detector working in the presence of colored noise is not as clean a problem as we have been discussing up until now. However, an interesting approximation to it is obtained by a first-order modification of Harris' result. If one assumes that the noise is colored but that the detector thinks that it is white, then we obtain the same expressions given in equation (16) \& (17) for the probabilities for T.P. and F.P., where now the signal to noise ratio becomes,

$$
d^{2}=d_{3}^{2}=\frac{\gamma^{2}\left[\int M_{w}^{2}(f) d f\right]^{2}}{\int N(f) \cdot M_{w}^{2}(f) d f}
$$

The authors nominate this as a candidate for an index of image quality (with modifications suggested in the next section) because of the intuitive appeal of the noise denominator when written in the spatial domain,

$$
N=\int d x \int d x^{\prime} \operatorname{Image}(x) \cdot C\left(x-x^{\prime}\right) \cdot \operatorname{Image}\left(x^{\prime}\right)
$$


This is a convolution or smearing of the image with the correlation function of the noise (F.T. of $N(f)$ ), overlapped with the image (analogous to a single scattering term in many branches of physics).

Under a different set of approximations than the above, Lawson (Ref. 47) has obtained the result

$$
d^{2}=d_{2}^{2}=\gamma^{2} \int \frac{M_{W}^{2}(f) d f}{N(f)}
$$

that is, the same signal-to-noise ratio as obtained with the matched filter discussed earlier. In fact, the complete likelihood detector can be implemented by generalized matched filters (Ref. 51). Thus, as with the matched filters, the likelihood detector is only troubled by noise in the neighborhood of the signal spectrum.

The signal-to-noise ratios considered above will be dimensionless quantities if the noise measurements are taken from density fluctuations. They represent two quasi-extremes hinted at earlier in equations (10) and (11), namely, the ratio of two integrals (equation (19)), or the integral of a ratio (equation (15)). In a moment we will return to the applicability of these models and the question of masking of signals by noise.

If we allow the decision criterion in the above analysis to vary on either side of unity, the $\mathrm{P}_{\mathrm{T}} . \mathrm{P}$. vs. $\mathrm{P}_{\mathrm{F}} \mathrm{P}$. curve traces out the so-called Receiver operating Characteristic (R.O.C.). This might correspond to changing the persistence of a radar screen or some analogous threshold setting in the case of an electronic signal detector (Ref. 52), or to varying the set, attitude, or motives of an observer who serves as an image signal detector (Ref. 53). This effect is displayed in Figure 8 where $x_{c}$ is proportional to the $\mathrm{log}$ of the decision criterion, and the other parameters can be calculated from signal and noise integrals as discussed above and in Refs. 47-49 (for the case of additive Gaussian noise). As one lowers his decision criterion he can score more true positives at the expense of more false alarms.

An example of an experimentally obtained R.O.C. is given in Figure 9. This is taken from the work of Goodenough et al. who studied the detectability of small low-contrast signals in radiographic images (Ref.46, 54). It is seen that the experimental curve is not symmetric about the negative diagonal, as the simple model above would predict.

This problem, some subjective and objective factors which affect the shape of R.O.C.

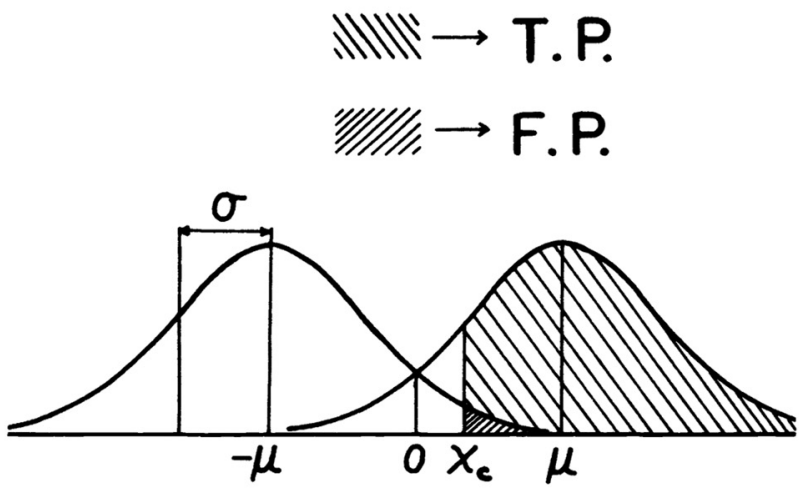

Figure 8. The effect of the decision threshold $\mathrm{x}_{\mathrm{c}}$ or "set" on the probabilities for true positive and false positive. As this is varied, a Receiver Operating Characteristic is generated.

curves, and methods of reducing the data of such curves are elaborated upon by Goodenough. For this review it is sufficient to point out that the intersection of the negative diagonal and the R.O.C. curve is a measure of the signal-to-noise ratio in the complete system of image receptor plus interpreter (viewer); that is, it is related to the parameter $\underline{d}$ introduced above. Correlation of studies with the various measures of signal-to-noise that have been proposed should help us to discover those models that are closest to reality.



Figure 9. R.O.C. curves obtained by Goodenough for an observer detecting small low contrast object radiographed with various screen-film combinations. The variation of the threshold is achieved by a rating procedure described by Goodenough (Ref. 46; courtesy of David J. Goodenough). 


\section{REAL DETECTORS}

The human detector does not quite attain the performance of the optimum detectors discussed above (Ref. 55). We have pointed out that in these systems only that noise in the neighborhood of the signal spectrum is effective in masking the signal. This seems to be a more reasonable model for applications to psychophysical studies of audition. The ear-brain system is capable of responding to temporal frequencies over a range of the order of 20,000 cycles per second. Yet, some experiments show that only a relatively narrow band of noise (of order $100 \mathrm{cps}$ ) centered about a signal of 1,000 cps is effective in masking that signal (Ref. 48). The size of this critical band and its effect upon complex signals is still unresolved.

The situation with the human eye-brain system and its selectivity in tuning for spatial frequencies has not yet been resolved. Some recent data from Greis and Röhler (Ref. 56) suggests that one-dimensional noise over a range of two octaves is effective in masking a signal consisting of a pure sinusoidal grating. When noise within this critical band is eliminated, a new and sharper curve of detectability is generated. See Figrures 10 and 11. These results are amplified in a study by Stromeyer \& Jules $s_{\mathbf{g}}$ in which dynamic one-dimensional noise within a band of approximately +1 octave was found to be effective in māsking sinusoidal gratings (Ref. 57).

This suggests that some convolution or smearing mechanism is at work in the frequency



Figure 10. Dependence of the signal amplitude ( $I$ to VI--dotted lines) of a sinusoidal grating needed for recognition, on the energy content of the interfering (masking) spectrum (band limits I to VI--solid lines). (Ref.56; courtesy of Optica Acta). domain, as if a spatial frequency were being averaged over a wide range. One mechanism for this might be the fall-off of the foveal vision off-axis: The retina acts as a data window (mentioned earlier in this paper). Unfortunately, although the order of magnitude of this smearing in the frequency domain is correct it is still too small to cover the effect. Perhaps the smearing comes about from limitations of the memory with respect to the spatial frequency it is trying to detect. Whatever the source of this spreading, it would be a straightforward matter to smear the integrands in the above expressions over a critical band by a moving average technique.

DeBelder et al. (Ref. 58) have tried to mock up the effect of a broad critical band by writing the signal-to-noise ratio as

$$
d^{2}=\frac{\gamma^{2} \int_{w}^{M_{w}^{2}}(f) \cdot E^{2}(f) d f}{\int N(f) \cdot E^{2}(f) d f+\Phi}
$$

Here $\Phi$ is internal noise generated within the eye, and the departure from ideal detection is made in two ways: the MTF of the eye is included explicitly as in earlier indexes; the critical band in which noise is effective as a mask is made to be as wide as the entire passband of the eye.

The true situation may not be quite this bad. Some recent work by Richards and Spitzberg (Ref. 59) suggests that we may have here a situation analogous to that in the perception of color. In that case three broad-band filters (the cone photopigments) together with neural interactions are capable of producing many narrow-band channels. Their work suggesta that in the domain of spatial-frequency

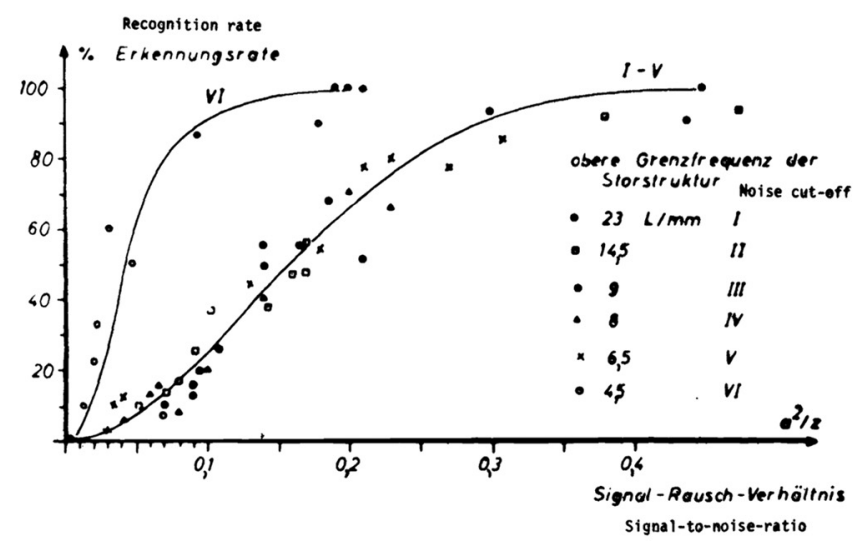

Figure 11. Dependence of the recognition rate of a sinusoidal grid masked by noise on the signal-to-noise energy ratio. The parameter ( $I$ to VI) designates the extent of the interfering spectrum (see Fig. 10) (Ref. 56; courtesy of Optica Acta.) 
analysis three broad-band spatial filters could produce narrow-band adaptation effects. This leans us back in the direction of treating the eye-brain as optimal or matched filter.

It is quite possible that the indexes discussed above may give roughly the same results. The signal-to-noise ratios reduce to the same expression when the noise is white. And if they are normalized to RMS contrasts (signal and noise) they reduce to expressions used in the perceptibility studies referenced earlier.

If calculations show great variations among the various indexes, perhaps correlations with other studies might help investigators in this field to determine if there exists an optimal index for a particular task. For example, hospital field studies that will ultimately be used in our investigation may indicate whether "a good radiograph is one that looks like other (good) radiographs" or if in fact the problem can be resolved quantitatively using image quality indexes.

We may learn how strong is the analogy between the eye and the ear. Perhaps it might be better to listen to radiographs. Then the ear will have to be trained, for it can be easily deceived as when it is made to think it is a nose by bad placement of stereo speakers (Ref. 60).

\section{ACKNOWLEDGEMENTS}

The authors gratefully acknowledge helpful discussions with D. Acheson, M. Cleare, E. Doerner, K. Doi, A. Haus, R. Holland, J. Jalickee, H. Kundel, N. Modine, C.N. Nelson, G.U.V. Rao, G. Revesz, K. Rossmann, G. Sanderson, W. Scherer, R. Schneider, F. Shea, J. Sullivan \& M. Ziskin.

We also express our appreciation for private communications and preprints received from several of the referenced authors.

\section{REFERENCES}

1. Buchanan, Robert A. An Improved X-ray Intensifying Screen. IEEE Trans. on Nucl. Sci. NS-19(1):81, (1972). An expanded version of this has appeared in Buchanan, R.A., Finkelstein, S.I., \& Wickersheim, K.A. X-ray Exposure Reduction Using Rare-Earth Oxysulfied, Intensifying Screens Radiology 105, 185 (1972).

2. Buchanan, R. A., Alves, R. V., Sobon, L. E., Bailey, H. N., Grench, H. A. Study of Rare-Earth Activated Materials for Radiation-
Sensing Applications Lockheed Missiles \& Space Company Document IMSC-D309853 Palo Alto, California (1972).

3. Bates, Lloyd M. Some Physical Factors Affecting Radiographic Image Quality: Their Theoretical Basis and Measurement, Public Health Service Publication No. 999-RH-38 (1969).

4. Haus, A. G., and Rossmann, K. X-ray Sensitometer for Screen-Film Combinations Used in Medical Radiology. Radiology 94, 673 (1970).

5. Rossmann, K., Lubberts, G., and Cleare, $H$. M. Measurement of the Line-SpreadFunction of Radiographic Systems Containing Fluorescent Screens. J. Opt. Soc. Am. 54, 187 (1964).

6. Rossmann, Kurt Point Spread-Function. Line Spread-Function, and Modulation Transfer Function. Radiology 93, 257 (1969).

7. Marchand, E. W. Derivation of the Point Spread Function from the Line Spread Function. J. Opt. Soc. Am. 54, 915 (1964).

8. Rossmann, Kurt Measurement of the Modulation Transfer Function of Radiographic Systems Containing Fluorescent Screens. Physics in Medicine and Biology 9, 551 (1964).

9. Morgan, R. H., Bates, I. M., Gapala Rao, U.V. and Marinaro, A. The Frequency Response Characteristics of X-ray Films and Screens. Am. J. Roentgenol. 92, 426 (1964).

10. Cleare, H. M., Splettstosser, H. R., and Seemann, H. E. Experimental Study of Mottle Produced by X-ray Intensifying Screens. Am. J. Roentgenol. 88, 168 (1962).

11. Rossmann, Kurt. Spatial Fluctuations of X-ray Quanta and the Recording of Radiographic Mottle. Am. J. Roentgenol. 90, 863 (1963).

12. Jones, R. Clark. New Method of Describing and Measuring the Granularity of Photographic Materials. J. Opt. Soc. Am. 45, 799 (1955).

13. Doerner, Edward C. Wiener-Spectrum Analysis of Photographic Granularity. J. Opt. Soc. Am. 52, 699 (1962).

14. Doi, Kunio. "Wiener Spectrum Analysis of Quantum Statistical Fluctuation and Other Noise Sources in Radiography" in "Television in Diagnostic Radiology", Mosely, Robert D. Jr., \& Rust. John H. (Eds.) Aesculapius Publishing Company, Birmingham, Alabama (1969) 
15. DeBelder, M. and DeKerf, J. The Determination of the Wiener Spectrum of Photographic Emulsion Layers with Digital Methods. Photographic Science and Engineering 11, 371 (1967).

16. Jenkins, Gwilym M., and Watts, Donald G. "Spectral Analysis and Its Applications" Holden-Day, San Francisco (1968).

17. Cooley, J. W., Lewis, P.A.W., Welch, P. D. "The Fast Fourier Transform Algorithm and Its Applications". IBM Research Paper RC-1743 Yorktown Heights, New York (1967)

18. Kolers, Paul A. "Some Psychological Aspects of Pattern Recognition" in "Recognizing Patterns (Kolers, P. \& Eden, M. Eds.) M.I.T. Press. Cambridge, Mass. (1967).

19. Kolers, Paul A., and Eden Murray (Eds.) Preface to Chung, Shin-Ho "Neurophysiology of the Visual System" in "Recognizing Patterns" M.I.T. Press. Cambridge, Mass. (1967).

20. Kühl, walter. A Critical Review of Methods of Image Evaluation - in Moseley, R. D. \& Rust, J. H. (Eds.) Television in Diagnostic Radiology. Aesculapius Publishing Co., Birmingham, Alabama (1969).

21. Rossmann, K. and Wiley, B. E. The Central Problem in the Study of Radiographic Image Quality. Radiology 96, 113 (1970).

22. Schade, Otto Sr. Optical and Photoelectric Analog of the Eye. J. Opt. Soc. Am. 46, 721 (1956).

23. Lowry, E. M., and DePalma, J. J. SineWave Response of the Visual System, I. The Mach Phenomenon. J. Opt. Soc. Am. 5l, 740 (1961).

24. Cornsweet, Tom N. "Visual Perception" Academic Press, New York and London (1970).

25. Kelley, D. H. Effects of Sharp Edges on the Visibility of Sinusoidal Gratings. J. Opt. Soc. Am. 60, 98 (1970). This paper contains eighteen references to work on the sine wave response of the eye.

26. Shea, F. J., and Ziskin, M. C. Visual System Transfer Function and Optimal Viewing Distance for Radiologists. Invest. Radiol. (in press) and Private Communication.

27. Ratliff, Floyd. Contour and Contrast. Scientific American June (1972)

28. Campbell, Fergus W. The Human Eye as an Optical Filter. Proceedings of the IEEE 56, 1009 (1968).
29. O'Neill, Edward L. "Introduction to Statistical Optics". Addison-Wesley

Publishing Co., Inc. Reading, Mass.

30. Linfoot, E. H. "Fourier Methods in Optical Image Evaluation". Focal Press London and New York (1964 English Edition).

31. Scade, Otto H., Image Gradation, Graininess and Sharpness in Television and Motion Picture Systems II. J. of the SMPTE 58, 181 (1952)

32. Higgins, G. C., and Jones, L. A. The Nature and Evaluation of the Sharpness of Photographic Images. J. Soc. Motion Picture T.V. Engrs. 58, 277 (1952).

33. Roetling, P. G., Trabka, E. A. \& Kinzly, R. E. Theoretical Prediction of Image Quality. J. Opt. Soc. Am. 58, 342 (1968).

34. Ooue, S. Progress in Optics. VII, 301 (1969) Wiley, Interscience.

35. Rao, Gopala U.V. Image Contrast of Sinusoidal Signals. Am. J. Roentgenology, Radium Therapy \& Nuclear Med. (to be published) and Private Communication.

36. Halmshaw, R. The Influence of Film Granularity on Image Detail on Radiographs. J. of Photographic Science 19, 167 (1971).

-37. Shannon, C. E. and Weaver W. "The Mathematical Theory of Communication". University of Illinois Press. Urbana (1949) (Shannon's Mean Power Thm).

38. Rose, Albert. The Sensitivity Performance of the Human Eye on an Absolute Scale. J. Opt. Soc. Am. 38, 196 (1948).

39. Coltman, J. W., and Anderson, A. E. Noise Limitations to Resolving Power in Electronic Imaging. Proceedings of the IRE 48, 858 (1960).

40. Legault, Richard R. Visual Detection Process for Electrooptical Images: Man-The Final Stage of an Electrooptical Imaging System. in Photoelectronic Imaging Devices, Vol. 1, Biberman, I. M. \& Nudelman, S. (Eds.) Plenum Press. New York-London (1971).

41. Moran, P. R. A Statistical Model for Perception of Objects in a Noisy Image. Proc. of Third Int. Conf. on Medical Physics Gotheburg, Sweden, August (1972) and Private Communication.

42. Morgan, R. H. Threshold Visual Perception and Its Relationship to Photon Fluctuation and Sine-Wave Response. Amer. J. Roentgenol. 92, 982 (1965) 
43. Rao, Gopala U. V. Contrast Perception in Imaging Systems. Int. J. of Applied Radiation and Isotopes 21, 571 (1970).

44. Andrews, Harry C. "Computer Techniques in Image Processing". Academic Press New York and London (1970).

45. Rindfleisch, T. and Willingham, D. A Figure of Merit Measuring Picture Resolution in "Advances in Electronics and Electron Physics"22A McGee et al.(Eds.) Academic Press, London and New York 341 (1966).

46. Goodenough, David J. Radiographic Applications of Signal Detection Theory . $\mathrm{Ph} . \mathrm{D}$. Thesis, University of Chicago (1972).

47. Lawson, Walter Electrooptical System Evaluation. in Photoelectronic Imaging Devices Vol. 1 Lucien M. Biberman \& Sol Nudelman (Eds.) Plenum Press, New York (1971).

48. Green, David M., and Swets, John A. "Signal Detection Theory and Psychophysics". John Wiley and Sons, Inc. New York (1966).

49. Harris, J. L. Resolving Power and Decision Theory. J. Opt. Soc. Am. 54, 606 (1964).

50. Cook, Charles E., and Bernfeld, Marvin "Radar Signals". Academic Press New York and London (1967).

51. Whalen, Anthony D. "Detection of Signals in Noise". Academic Press New York and London (1971).

52. Peterson, W. W., Birdsall, T. G., and Fox, W. C. The Theory of Signal Detectability. Trans. IRE Professional Group on Information Theory PGIT-4, 171, (1954).
53. Lusted, Lee B. "Introduction to Medical Decision Making". Charles C. Thomas, Springfield, Illinois (1968).

54. Goodenough, David J., Rossman, Kurt, and Lusted, Lee B. Radiographic Applications of Signal Detection Theory. Radiology, 105, 199, (1972).

55. Stevens, S. S. A Neural Quantum in Sensory Discrimination. Science 177, 749, (1972). The curves contained in this article and those contained in Refs. 41,56 make an interesting comparison.

56. Greis, U. und Röhler, R. Untersuchung der subjektiven Detailerkennbarkeit mit Hilfe der Ortsfrequenzfilterung Optica Acta 17, 515 (1970). (Also the English translation of this article by Normal Modine of the Bureau of Radiological Health).

57. Stromeyer, Charles F. III \& Julesz, Bela Spatial-Frequency Masking in Vision: Critical Bands and Spread of Masking. J. Opt. Soc. Am. 62, 1221 (1972) .

58. DeBelder, M., Bollen, R., \& Duville, R. A New Approach to the Evaluation of Radiographic Systems. The Journal of Photographic Science 19, $126(1 \overline{971)}$

59. Richards, Whitman \& Spitzberg, Richard Spatial-Frequency Channels: Many or Few? 1972 Annual Meeting - Optical Society of America San Francisco (1972).

60. Allen, Woody "Getting Even". Random House New York (1971).

61. Rossmann, Kurt. Some Physical Factors Affecting Image Quality in Medical Radiography. J. Photographic Science 12, 279, (1964).

\section{THE AUTHORS}

ROBERT F. WAGNER is doing Image Analysis with the Bureau of Radiological Health. His publications have dealt with pion, electron, and photon interactions. Dr. Wagner holds an M.A. from Augustinian College and his M.S. and Ph.D. in Nuclear Physics from Catholic University. He also held a NASA Pre-Doctoral Fellowship, NATO summer Fellowships abroad, and an NSF Post-Doctoral at Ohio University.

KENNETH E. WEAVER is a Research Physicist at the Bureau of Radiological Health, serving as Chief of the Standards Development Section of the X-Ray Exposure Control Laboratory. Mr. Weaver received his B.S. in Physics (1966) from California State College at Los Angeles and his M.S. in Radiological Physics (1968) from Oregon State University.

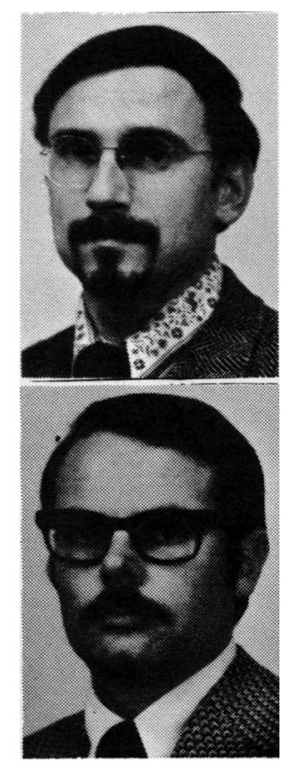

